\title{
RESEARCH
}

Open Access

\section{Splenic enlargement induced by preoperative chemotherapy is a useful indicator for predicting liver regeneration after resection for colorectal liver metastases}

\author{
Takanori Konishi ${ }^{1}$, Hiroyuki Yoshidome ${ }^{1,2^{*}}$, Hiroaki Shimizu ${ }^{1}$, Hideyuki Yoshitomi ${ }^{1}$, Katsunori Furukawa ${ }^{1}$,
} Tsukasa Takayashiki', Satoshi Kuboki', Shigetsugu Takano', Masaru Miyazaki ${ }^{1,3}$ and Masayuki Ohtsuka'

\begin{abstract}
Background: Conversion chemotherapy may downsize unresectable colorectal liver metastases (CRLMs), but may cause liver injury and splenic enlargement. The effect of preoperative chemotherapy on liver regeneration after liver resection remains undetermined. The aim of this study was to examine whether splenic enlargement induced by preoperative chemotherapy is an indicator to identify high-risk patients for impaired liver regeneration and liver dysfunction after resection.

Methods: We retrospectively reviewed 118 Japanese patients with CRLMs. Fifty-one patients had conversion chemotherapy. The other 67 patients underwent up-front liver resection. We clarified effects of conversion chemotherapy on splenic volume, liver function, and postoperative liver regeneration. Perioperative outcome was also analyzed.

Results: A ratio of the splenic volume before and after chemotherapy (SP index) in the oxaliplatin-based chemotherapy group was significantly greater than other chemotherapy groups after 9 or more chemotherapy cycles. Patients whose SP index was 1.2 or more had significantly higher indocyanine green retention rate at 15 min (ICG-R15) than patients without chemotherapy. Analyses of covariance showed liver regeneration rate after resection was decreased in patients whose SP index was 1.2 or more. The incidence of postoperative liver dysfunction in patients whose SP index was 1.2 or more was significantly greater than patients without chemotherapy. Multivariate analysis showed SP index was a significant predictive factor of impaired liver regeneration.

* Correspondence: h-yoshidome@umin.ac.jp

'Department of General Surgery, Chiba University Graduate School of

Medicine, 1-8-1 Inohana, Chuo-Ku, Chiba 260-8670, Japan

${ }^{2}$ Department of Surgery, Oami Municipal Hospital, 884-1 Tomida, Oami-Shirasato-shi, Chiba 299-3221, Japan

Full list of author information is available at the end of the article

C The Author(s). 2020 Open Access This article is licensed under a Creative Commons Attribution 4.0 International License, which permits use, sharing, adaptation, distribution and reproduction in any medium or format, as long as you give appropriate credit to the original author(s) and the source, provide a link to the Creative Commons licence, and indicate if changes were made. The images or other third party material in this article are included in the article's Creative Commons licence, unless indicated otherwise in a credit line to the material. If material is not included in the article's Creative Commons licence and your intended use is not permitted by statutory regulation or exceeds the permitted use, you will need to obtain permission directly from the copyright holder. To view a copy of this licence, visit http://creativecommons.org/licenses/by/4.0/. The Creative Commons Public Domain Dedication waiver (http://creativecommons.org/publicdomain/zero/1.0/) applies to the data made available in this article, unless otherwise stated in a credit line to the data. 
(Continued from previous page)

Conclusions: Splenic enlargement induced by preoperative chemotherapy was a useful indicator for impaired liver regeneration after resection and a decision-making tool of treatment strategy for unresectable CRLMs.

Keywords: Hepatectomy, Splenomegaly, Conversion chemotherapy, Colorectal liver metastases, Liver regeneration,

\section{Background}

Hepatic resection is a possibly curative treatment for colorectal liver metastases (CRLMs). However, up to $80 \%$ of CRLMs are initially diagnosed as unresectable [1]. Currently, 5-fluorouracil and folinic acid in combination with either oxaliplatin or irinotecan are commonly used cytotoxic regimens for colorectal cancer [2]. Biologics such as anti-VEGF and anti-EGFR monoclonal antibody are often used simultaneously for patients with unresectable colorectal cancer, as they improve the response and resection rate [3-5]. Thus, recent advances in chemotherapy for colorectal cancer may render unresectable CRLMs amenable to resection. Adam et al. demonstrated that conversion chemotherapy may downsize unresectable CRLMs [6]. They proceeded to hepatic resection in $12.5 \%$ of patients with initially unresectable CRLM, with a 5-year survival rate after liver resection of $33 \%$. However, preoperative chemotherapy causes liver injury, such as sinusoidal obstructive syndrome or steatohepatitis, which may affect postoperative outcomes after liver resection [7]. Sinusoidal obstruction syndrome associated with oxaliplatin is reported to cause an increased risk of postoperative morbidity and long hospital stay in patients undergoing major hepatectomy [8]. Sinusoidal damage is likely to elevate portal venous pressure, which may cause enlargement of the spleen. Therefore, an increase in the splenic volume is reported to be a predictive marker of the development of sinusoidal obstruction syndrome [9].

Liver regeneration is an important phenomenon to maintain liver volume and function after loss of liver parenchyma, such as with liver transplantation and hepatic resection. Chronic hepatitis and cirrhosis are associated with poor postoperative liver regeneration [10], and insufficient regeneration after major hepatectomy is likely to lead to liver failure [11]. Although chemotherapy is sometimes used before hepatectomy for CRLMs, the association between preoperative chemotherapy and liver regeneration after hepatic resection has not been well defined. It may be important to identify patients who will have insufficient liver regeneration and postoperative liver failure before operation. We hypothesized that preoperative chemotherapy may increase splenic volume, which might be associated with compromised liver regeneration. The objective of this study was to investigate splenic enlargement induced by preoperative chemotherapy for CRLMs as a marker for selecting the patients with impaired liver regeneration after liver resection.

\section{Methods}

\section{Patients and preoperative evaluation}

We retrospectively analyzed the medical records of 118 Japanese patients with resectable, not optimally resectable, or initially unresectable CRLM seen at the Department of General Surgery at Chiba University (Chiba, Japan) between 2005 and 2013. All patients were evaluated preoperatively with abdominal ultrasonography, thoracoabdominal dynamic multidetector-row computed tomography (MD-CT), and magnetic resonance imaging. The remnant liver functional reserve was predicted from the indocyanine green retention rate at $15 \mathrm{~min}$. The future remnant liver volume was predicted by CT volumetry in every patient for whom liver resection was planned. Patients with a predicted remnant liver volume $\geq 35 \%$ of the total liver volume underwent liver resection without preoperative portal vein embolization or staged hepatectomy. The resectability of each patient was evaluated based on modification of the previous report [12]. Briefly, patients whose predicted remnant liver volume is far less than $35 \%$ of the total liver because of biloblar multiple tumors or the invasion or close to all of the hepatic veins or both portal branches were defined as initially unresectable CRLM. Patients who have poor prognostic factors such as extrahepatic metastases were defined as not optimally resectable CRLM.

Of the 118 patients analyzed, 51 received conversion chemotherapy for not optimally resectable or initially unresectable CRLMs. Chemotherapy regimen was chosen based on the guideline from Japanese Society for cancer of the Colon and Rectum. Second-line regimen chemotherapy was performed when first-line regimen was not effective. Hepatic resection was proceeded when unresectable CRLMs were converted to technically resectable by conversion chemotherapy. The other 67 patients underwent liver resection without preoperative chemotherapy during the same period. All patients have given informed consent in accordance with the ethical standards. The study protocol has been approved by the institute's committee on human research. The approval code obtained from the corresponding ethical committee on human research was \#3150 (Chiba University School of Medicine). We retrospectively analyzed details of the metastatic tumors, operative data, and postoperative 
complications as defined by the Clavien-Dindo classification [13]. Steatosis was diagnosed when liver attenuation was less than or equal to spleen attenuation minus $10 \mathrm{HU}$ in unenhanced CT according to the previous report [14].

\section{Splenic volume and liver volume analysis}

For patients who received chemotherapy, an enhanced MD-CT was performed before and after chemotherapy in order to assess the indications for hepatic resection. The splenic volume before (pre-SP) and after chemotherapy (post-SP) was measured in an identical manner by MD-CT. A change in the splenic volume during chemotherapy (SP index) was calculated as the ratio of post-SP to pre-SP. The preoperative entire liver volume (ELV) exclusive of CRLMs and the preoperative future remnant liver volume (FRLV) were measured by MDCT. The ratio of FRLV to ELV was calculated as $100 \times$ FRLV/ELV. CT was performed in patients 7 days after liver resection to assess postoperative complications and calculate the remnant liver volume $\left(\operatorname{RLV}_{7}\right)$. The liver regeneration rate was defined as $\mathrm{RLV}_{7} /$ FRLV. Splenic and liver volumes were measured by SYNAPSE VINCENT (Fuji Photo Film CO., Ltd. Japan).

\section{Liver regeneration analysis}

In order to identify whether chemotherapy-induced liver damage affected liver regeneration after hepatectomy, the cutoff value of the SP index was defined by the receiver-operating characteristic (ROC) curve. According to this value, we divided the patients into three groups. Characteristics of the patients, operative data, liver regeneration rate, and postoperative course were analyzed in these three groups.

\section{Statistical analysis}

Summary statistics were constructed for baseline values, employing frequencies and percentages for categorical data and means and standard deviations for continuous variables. We compared patient characteristics using Fisher's exact test for categorical outcomes, MannWhitney $U$ test for continuous unpaired outcomes, and Wilcoxon signed-rank test for continuous paired outcomes. We compared the patients' data among the three groups by using the chi-square test for categorical outcomes and the Kruskal-Wallis test for continuous outcomes. The relationship between a change in splenic volume and liver regeneration was assessed by using analysis of covariance to adjust for imbalances in the ratio of FRLV to ELV. Multivariate analysis was performed using the logistic regression model. A $p$ value of $<0.05$ (two-tailed tests) was considered to be significant. All statistical analyses were performed using the SPSS 18.0 software program (Chicago, IL).

\section{Results}

\section{Patients and chemotherapy}

Among the 51 patients who received chemotherapy, 43 were able to proceed to hepatectomy, while 8 still had unresectable CRLMs after chemotherapy. The other 67 patients underwent up-front hepatectomy. The numbers of liver metastases, patients with positive lymph node metastases of the primary lesion, and patients with extrahepatic metastases in those treated with chemotherapy were significantly greater than in those without chemotherapy. Of those receiving chemotherapy, $45 \mathrm{pa}-$ tients had a first-line regimen and 6 had a second-line regimen. Oxaliplatin-based chemotherapy was administered in 29 patients and irinotecan-based therapy in 20 patients. Fluorouracil plus leucovorin was given to 2 patients. The median chemotherapy course was 9 cycles, and 27 patients received 9 cycles or more.

\section{Changes in splenic volume}

To determine whether chemotherapy affects splenic volume, volumetric analyses of the spleen before and after chemotherapy were performed. The splenic volume significantly increased after chemotherapy (Fig. 1a, $p=$ 0.036). To assess whether splenic volume was affected by specific chemotherapy regimens, we divided patients into three groups stratified by regimen: FOLFIRI/IRIS with or without targeted therapies (IRI-based, $n=20$ ), FOLFOX/CapeOX with or without anti-EGFR monoclonal antibodies (OX-based, $n=18$ ), and FOLFOX/ CapeOX with bevacizumab (OX-based + Bmab, $n=11$ ). The two patients who received fluorouracil + leucovorin were excluded in this analysis. The SP index in the OXbased group was significantly greater than that of the IRI-based group (Fig. 1b, $p=0.018$ ). Among patients who underwent 9 or more chemotherapy cycles, the SP index of the OX-based group was significantly greater than that of IRI-based and OX-based + Bmab groups (Fig. 1c, $p=0.008,0.007$, respectively). There was no significant difference among patients receiving 8 or fewer cycles of chemotherapy (Fig. 1d). Here, we show a representative case of chemotherapy-induced splenomegaly. A patient underwent 20 cycles of OX-based chemotherapy before liver resection. The splenic volume increased during the chemotherapy (Fig. 2a; SP index =1.34), and the histological analysis showed severe sinusoidal obstruction syndrome in the resected liver specimen after resection (Fig. 2b).

\section{Liver regeneration and postoperative course after hepatectomy}

To determine whether preoperative chemotherapyinduced splenomegaly may affect liver regeneration after hepatectomy, we defined the cutoff value of the SP index by the ROC curve as 1.2. We divided the patients who 
a

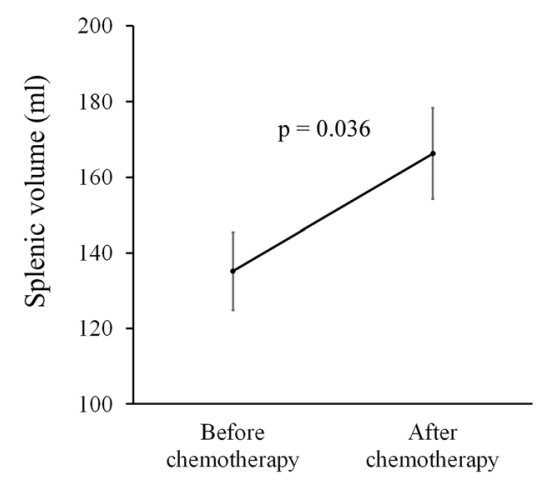

C

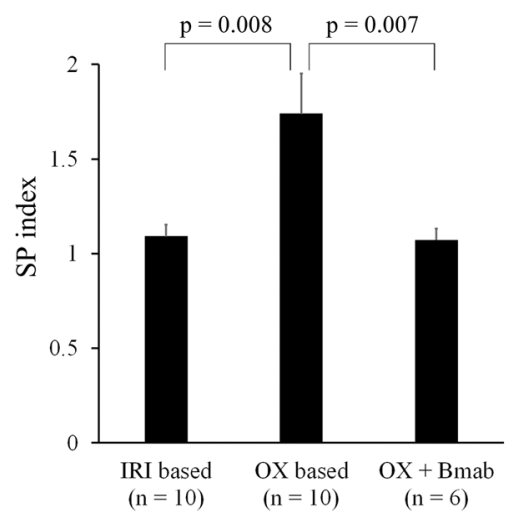

$\mathrm{b}$

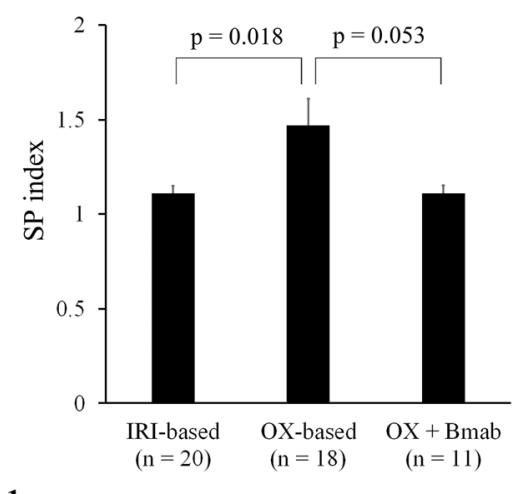

$\mathrm{d}$

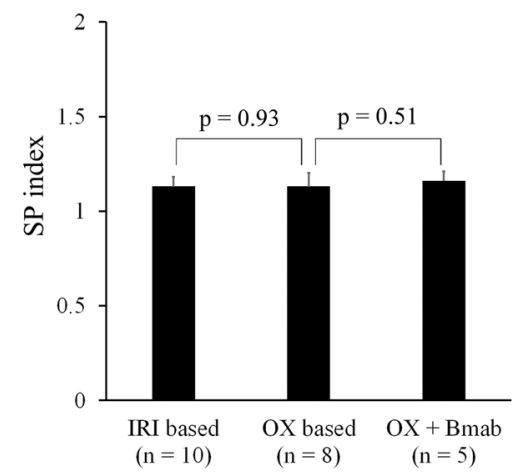

Fig 1 Changes in splenic volume during chemotherapy. a Splenic volume before and after preoperative chemotherapy $(n=51)$. b The relationship between SP index and chemotherapeutic regimen. IRI-based: FOLFIRI/IRIS with or without biologics $(n=20)$. OX-based: FOLFOX/ CapeOX with or without anti-EGFR monoclonal antibodies $(n=18)$. OX + Bmab: FOLFOX/CapeOX with bevacizumab $(n=11)$. c SP index in patients who had 9 or more cycles of chemotherapy. $\mathbf{d}$ SP index in patients who had 8 or fewer cycles of chemotherapy. Data are mean \pm standard error of the mean. A comparison was performed using the Mann-Whitney $U$ test. A $p$ value $<0.05$ was considered to be significant

underwent hepatectomy into three groups: those receiving chemotherapy and whose SP index was 1.2 or more $(n=16)$, those receiving chemotherapy and whose SP index was less than $1.2(n=27)$, and those without preoperative chemotherapy $(n=67)$. Table 1 shows patient characteristics in each group. There were no significant differences in sex, age, body mass index (BMI), prevalence of diabetes mellitus, hepatic virus infection, or steatosis in the three groups. The ICG-R15 in patients whose SP index was 1.2 or more was significantly greater than in patients without preoperative chemotherapy. Analyses of covariance showed that the liver regeneration rate in patients whose SP index was 1.2 or more was significantly lower than in patients whose SP index was less than 1.2 and in patients without preoperative chemotherapy, adjusting for imbalances of the ratio of FLRV to ELV (Fig. 3a, $p=0.021,0.033$, respectively), but ICG-R15 did not significantly affect liver regeneration in this set of the patients (Fig. 3b, $p=0.40$ ).

To identify the factors that affect liver regeneration after hepatectomy and to determine whether changes in splenic volume influence the operative and postoperative course, we selected a subset of patients undergoing major hepatectomy (resection of three or more segments in Couinaud's classification) $(n=56)$. The patients' characteristics are shown in Table 2. The splenic volume and levels of serum aspartate and alanine aminotransferase before hepatectomy in patients whose SP index was 1.2 or more $(n=12)$ were significantly greater than in those whose SP index was less than $1.2(n=14)$ and in patients without preoperative chemotherapy $(n=30)$. Platelet counts before hepatectomy in patients whose SP index was 1.2 or more were significantly lower than in patients without chemotherapy and serum albumin levels before hepatectomy in patients whose SP index was 1.2 or more were significantly greater than in patients without chemotherapy. We analyzed factors affecting liver regeneration after hepatectomy with logistic and multiple linear regression, adjusting for possible differences in baseline scores and background characteristics such as sex, age (more than 70 years old), BMI (more than 25), diabetes mellitus, number of 
a

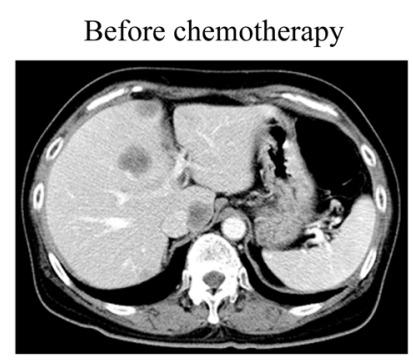

$\mathrm{b}$

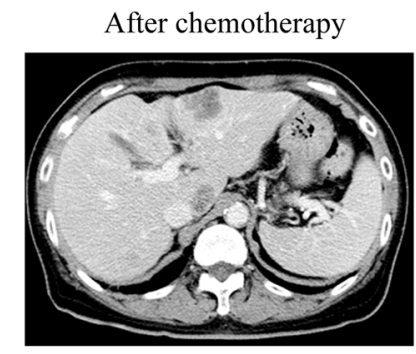

C

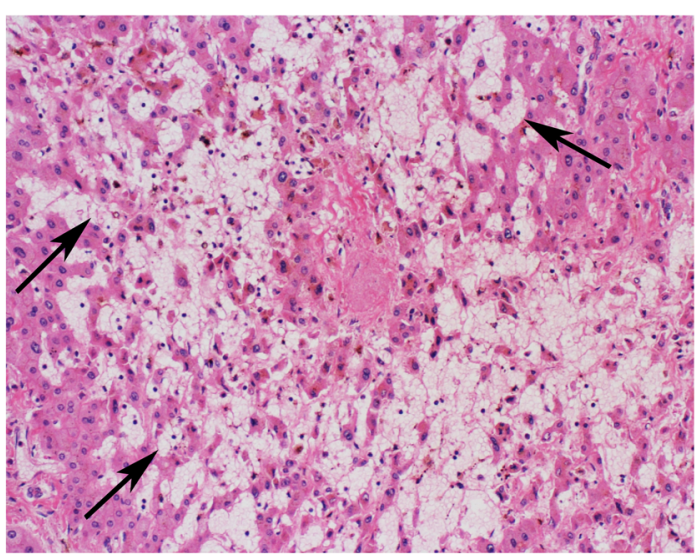

Fig 2 Representative case of splenomegaly induced by chemotherapy. a Abdominal dynamic multidetector-row computed tomography (MD-CT) findings before chemotherapy. b MD-CT findings after 20 cycles of oxaliplatin-based chemotherapy. The splenic volume increased during chemotherapy (SP index = 1.34). c Histological analysis (hematoxylin and eosin staining) of the liver after resection. The non-tumoral liver developed sinusoidal obstruction syndrome. Arrows indicate dilatation of sinusoids. Original magnification is $\times 200$

chemotherapy cycles ( 9 cycles or more), SP index (1.2 or more), platelet counts before operation (more than the lower limit of normal), and concomitant portal vein embolization. The SP index was a significant factor predictive of impaired liver regeneration on multivariate analysis (odds ratio, 7.889; 95\% CI, $1.132 \sim 54.961 ; p=$ 0.037).
Surgical data and postoperative events are shown in Table 3. Operation time and blood loss during surgery in patients whose SP index was 1.2 or more were significantly greater than in patients without preoperative chemotherapy. We investigated the incidence of postoperative liver failure in the three groups. This was defined as hyperbilirubinemia (at least twice the upper limit of

Table 1 Characteristics of patients undergoing liver resection for colorectal liver metastases

\begin{tabular}{|c|c|c|c|c|}
\hline \multirow[t]{2}{*}{ Characteristics } & \multicolumn{2}{|l|}{ Chemotherapy } & \multirow{2}{*}{$\begin{array}{l}\text { No } \\
\text { chemotherapy } \\
(n=67)\end{array}$} & \multirow{2}{*}{$\begin{array}{l}p \\
\text { value }\end{array}$} \\
\hline & SP index $\geq 1.2(n=16)$ & SP index $<1.2(n=27)$ & & \\
\hline Gender (male/female) & $11 / 5$ & $15 / 12$ & $35 / 32$ & 0.49 \\
\hline Age & $57.8 \pm 13.3$ & $62.1 \pm 10.3$ & $64.4 \pm 9.5$ & 0.10 \\
\hline BMI & $23.5 \pm 3.6$ & $21.5 \pm 2.9$ & $22.8 \pm 3.9$ & 0.14 \\
\hline Diabetes mellitus (+) & 4 & 5 & 8 & 0.38 \\
\hline Hepatitis virus $(+)$ & 0 & 0 & 2 & 0.52 \\
\hline Number of chemotherapy cycles (median, range) & $12(5-27)$ & $8(1-43)$ & - & 0.27 \\
\hline ICG-R15 (\%) & $13.5 \pm 9.5$ & $9.4 \pm 4.8$ & $8.6 \pm 4.8$ & 0.036 \\
\hline Steatosis before chemotherapy & 1 & 1 & - & 1.00 \\
\hline Steatosis before operation & 3 & 3 & 3 & 0.14 \\
\hline
\end{tabular}

Continuous data are presented as mean \pm standard deviation

$B M I$ body mass index, ICG-R15 indocyanin green retention rate at $15 \mathrm{~min}$ 
a

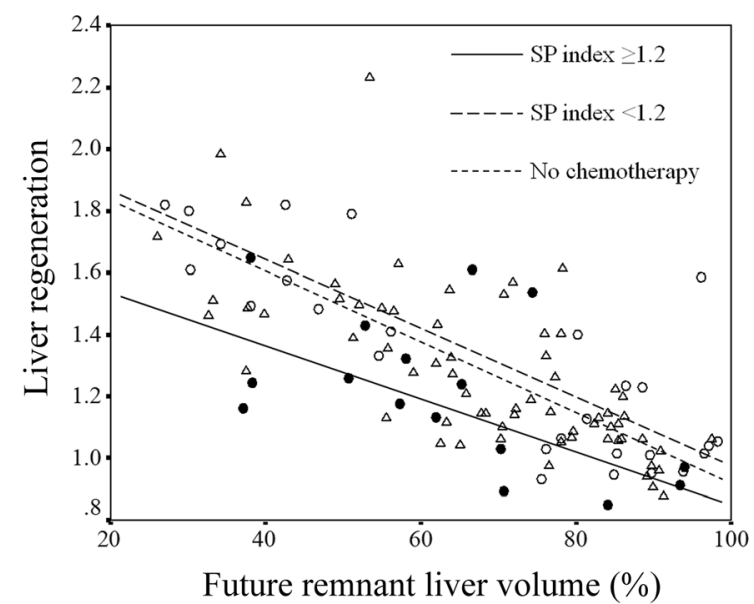

b

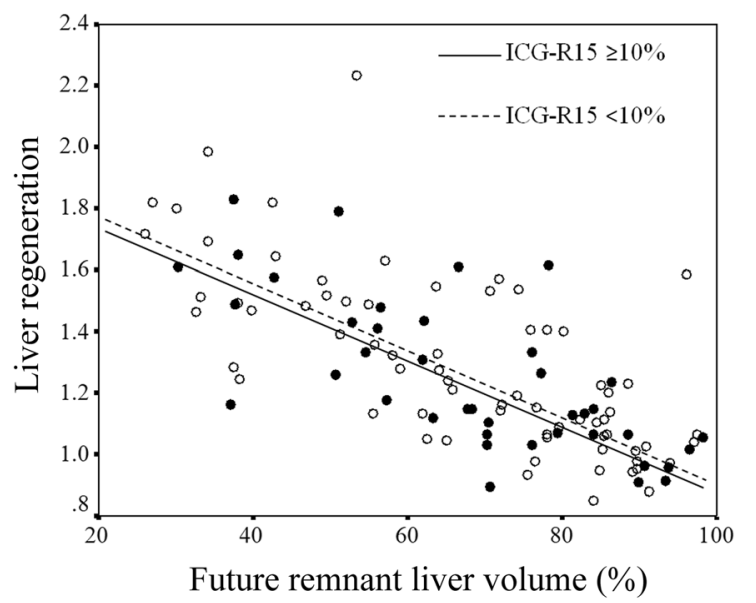

Fig 3 Relationship between splenic enlargement and liver regeneration. a The relationship between splenic enlargement and liver regeneration. SP index $\geq 1$.2: changes in splenic volume during chemotherapy were 1.2 or more $(\bullet, n=16)$. SP index $<1.2$ : changes in splenic volume during chemotherapy were less than $1.2(0, n=27)$. No chemotherapy: patients received no preoperative chemotherapy before hepatectomy $(\Delta, n=$ 67). There was statistical significance among these groups (SP index $\geq 1.2$ vs SP index $<1.2$ or no chemotherapy; $p=0.021,0.033$, respectively) defined by analyses of covariance. $\mathbf{b}$ The relationship between ICG-R15 and liver regeneration. ICG-R15 $\geq 10 \%(\bullet, n=40)$, ICG-R15 $<10 \%(0, n=$ 70). There was no significant difference in liver regeneration between the two groups $(p=0.40)$

Table 2 Characteristics and preoperative data of patients undergoing major hepatectomy

\begin{tabular}{|c|c|c|c|c|}
\hline \multirow[t]{2}{*}{ Characteristics } & \multicolumn{2}{|l|}{ Chemotherapy } & \multirow{2}{*}{$\begin{array}{l}\text { No } \\
\text { chemotherapy } \\
(n=30)\end{array}$} & \multirow[t]{2}{*}{$p$ value } \\
\hline & SP index $\geq 1.2(n=12)$ & SP index $<1.2(n=14)$ & & \\
\hline Gender (male/female) & $8 / 4$ & $8 / 6$ & $14 / 16$ & 0.48 \\
\hline Age & $58.3 \pm 12.4$ & $63.6 \pm 10.3$ & $63.0 \pm 10.0$ & 0.38 \\
\hline BMI & $22.9 \pm 3.1$ & $21.3 \pm 2.7$ & $22.8 \pm 3.8$ & 0.23 \\
\hline Diabetes mellitus (+) & 4 & 3 & 5 & 0.49 \\
\hline Hepatitis virus $(+)$ & 0 & 0 & 2 & 0.41 \\
\hline Number of chemotherapy cycles (median, range) & $12(5-27)$ & $8(1-23)$ & - & 0.21 \\
\hline SP index & $1.65 \pm 0.60$ & $1.04 \pm 0.05$ & - & $<0.001$ \\
\hline Splenic volume before chemotherapy & $131.7 \pm 48.2$ & $112.4 \pm 49.4$ & - & 0.23 \\
\hline Splenic volume before hepatectomy & $214.3 \pm 88.8$ & $117.7 \pm 56.9$ & $160.9 \pm 162.0$ & 0.004 \\
\hline \multicolumn{5}{|l|}{ Laboratory tests before hepatectomy } \\
\hline Platelet count $\left(\times 10^{9} / \mathrm{L}\right)$ & $171 \pm 60$ & $231 \pm 97$ & $234 \pm 72$ & 0.029 \\
\hline AST (IU/L) & $33.3 \pm 11.7$ & $24.8 \pm 7.8$ & $26.7 \pm 9.3$ & 0.035 \\
\hline ALT (IU/L) & $30.5 \pm 18.9$ & $19.2 \pm 13.6$ & $22.2 \pm 13.5$ & 0.032 \\
\hline ALP (IU/L) & $371.6 \pm 161.3$ & $410.9 \pm 186.3$ & $303.8 \pm 159.2$ & 0.050 \\
\hline Albumin (g/dL) & $3.9 \pm 0.3$ & $3.9 \pm 0.4$ & $4.1 \pm 0.2$ & 0.014 \\
\hline T-Bil (mg/dL) & $0.9 \pm 0.4$ & $0.9 \pm 0.4$ & $0.9 \pm 0.3$ & 0.94 \\
\hline PT-INR & $1.02 \pm 0.08$ & $1.04 \pm 0.09$ & $1.08 \pm 0.10$ & 0.17 \\
\hline ICG-R15 (\%) & $12.3 \pm 5.9$ & $10.2 \pm 6.0$ & $7.9 \pm 3.9$ & 0.061 \\
\hline Future remnant liver volume (\%) & $55.9 \pm 12.8$ & $49.3 \pm 18.5$ & $53.5 \pm 13.8$ & 0.42 \\
\hline TSH/PVE & 3 & 4 & 2 & 0.12 \\
\hline
\end{tabular}

Continuous data are presented as mean \pm standard deviation

$B M I$ body mass index, AST aspartate aminotransferase, $A L T$ alanine aminotransferase, $A L P$ alkaline phosphatase, $T$-Bil total bilirubin, $P T$-INR prothrombin time international normalized ratio, ICG-R15 indocyanine green retention rate at $15 \mathrm{~min}$, TSH two stage hepatectomy, PVE portal vein embolization 
Table 3 Operative and postoperative outcome after major hepatectomy

\begin{tabular}{|c|c|c|c|c|}
\hline & \multicolumn{2}{|l|}{ Chemotherapy } & \multirow{2}{*}{$\begin{array}{l}\text { No } \\
\text { chemotherapy } \\
(n=30)\end{array}$} & \multirow{2}{*}{$\begin{array}{l}p \\
\text { value }\end{array}$} \\
\hline & SP index $\geq 1.2(n=12)$ & SP index $<1.2(n=14)$ & & \\
\hline Operation time (min) & $388(255-551)$ & $306(260-739)$ & $289(190-430)$ & 0.035 \\
\hline Blood loss (ml) & $1368(225-3420)$ & $661(335-2560)$ & $570(285-4935)$ & 0.024 \\
\hline Intraoperative transfusion & $6(50 \%)$ & $7(50 \%)$ & $7(23.3 \%)$ & 0.12 \\
\hline Postoperative hyperbilirubinemia & $6(50 \%)$ & $4(28.6 \%)$ & $4(13.3 \%)$ & 0.043 \\
\hline Morbidity (Clavien-Dindo classification IIIミ) & $2(16.7 \%)$ & $3(21.4 \%)$ & $3(10 \%)$ & 0.58 \\
\hline Biliary leakage & 2 & 2 & 3 & \\
\hline Intra-abdominal abscess & 1 & 2 & 1 & \\
\hline Mortality & 0 & 0 & 0 & \\
\hline
\end{tabular}

Continuous data are presented as median and range

normal) on or after postoperative day 5 , according to the classification of the International Study Group of Liver Surgery [15]. The incidence of postoperative liver failure in patients whose SP index was 1.2 or more was significantly greater than in patients without chemotherapy (Table 3). On the other hand, there was no significant difference between patients whose SP index was less than 1.2 and those without chemotherapy.

\section{Discussion}

Recent advances in chemotherapy for colorectal cancer have improved the resection rate and prolonged survival for patients with initially unresectable CRLMs [1, 16-18]. Although several advantages of preoperative chemotherapy for CRLMs have been demonstrated, drawbacks have also been recognized $[19,20]$. Cytotoxic agents cause certain types of hepatic parenchymal injury, such as steatohepatitis or sinusoidal obstruction syndrome $[7,21]$, but the actual effect of chemotherapy-induced hepatotoxicity on liver regeneration after resection and postoperative complications remains unclear. This study revealed that an increase in splenic volume mediated by preoperative chemotherapy is associated with poor preoperative liver function, which is consistent with the previous report that showed oxaliplatin-based chemotherapy increases splenic volume as a result of sinusoidal injury [9]. Furthermore, we clarified that patients with splenic enlargement during chemotherapy had impaired liver regeneration and liver dysfunction after liver resection.

Long-term chemotherapy reportedly fails to improve the response rate, but it increases the incidence of sinusoidal injury, thus leading to postoperative liver failure [22]. In the current study, among patients undergoing 9 or more cycles of chemotherapy, splenomegaly in the OX-based group was significantly greater than that in the IRI-based and OX-based + Bmab-treated groups, which suggests that long-term oxaliplatin-based chemotherapy favorably causes splenic enlargement. Consistent with previous reports [23, 24], the current study also demonstrated that bevacizumab is likely to inhibit enlargement of the spleen, probably because of a decrease in sinusoidal injury. Patients with splenic enlargement had increased levels of aspartate aminotransferase and alanine aminotransferase and worse ICG-R15 those of which relate to liver injury and deterioration of hepatic function. Taken together, the evidence suggests that changes in splenic volume, which can be easily assessed before hepatectomy without invasive examination, are likely to be a marker of chemotherapy-induced liver damage and a decision-making marker of selecting chemotherapy regimen for conversion surgery.

The influence of preoperative chemotherapy on liver regeneration after liver resection is still controversial. Although some reports showed that preoperative chemotherapy itself does not affect liver regeneration and postoperative course [25-27], few studies have argued the adverse effects of chemotherapy-induced hepatotoxicity on early liver regeneration after liver resection. Current study suggested that splenic enlargement which represents liver injury caused by chemotherapy is related to impaired liver regeneration after hepatectomy. Our results also showed that there was no significant difference in liver regeneration between patients whose SP index was less than 1.2 and patients without preoperative chemotherapy. By measuring splenic volume during preoperative chemotherapy, we may preoperatively select the patients who will suffer chemotherapy-mediated impaired liver regeneration after resection.

Liver regeneration is achieved through multiple signaling pathways by mitogenic growth factors, such as HGF, EGF, and TGF- $\alpha$, leading eventually to hepatocyte growth and proliferation [28]. Sinusoidal endothelial cells play an important role in the release of HGF through VEGFR2 [29]. We measured liver volume 7 days after hepatectomy to assess early liver regeneration, which may directly correlate with postoperative liver failure. In the current study, patients with an SP index $\geq 1.2$ had less early liver regeneration after adjusting for 
imbalances of the FRLV, but there was no correlation between early liver regeneration and ICG-R15 as previously reported [30]. These results suggested that splenic enlargement is an indicator of liver regeneration after resection, while ICG-R15 represents hepatic functional reserve. Splenic volume is negatively correlated with liver regeneration after major hepatectomy [31]. Liver regeneration may be inhibited by splenic upregulation of TGF- $\beta 1$ and downregulation of HGF $[32,33]$. These findings suggest that a prolonged duration of OX-based chemotherapy that induces splenomegaly may affect liver regeneration. Furthermore, the sinusoidal obstruction syndrome model induced by monocrotaline resulted in decreased mRNA expression of mitogenic growth factors and impaired hepatic regeneration in rats [34]. Sinusoidal obstruction syndrome is likely to inhibit liver hypertrophy after first hepatectomy with portal vein embolization in a two-stage hepatectomy [35]. It seems likely that splenic enlargement induced by long-term preoperative chemotherapy influences liver regeneration after resection for CRLMs. Consistent with these findings and based on our multivariate analysis, an SP index of $\geq 1.2$ is a possible candidate marker for predicting impaired liver regeneration.

A previous study showed that sinusoidal injury increased the risk of greater morbidity and longer hospital stay after major hepatectomy [8]. An increase in splenic volume has been correlated with major complications after hepatic resection [36]. Current study showed that splenic enlargement was associated with a longer operative time and increased blood loss during operation. Furthermore, patients with an SP index $\geq 1.2$ were likely to be at greater risk of postoperative liver failure as defined by hyperbilirubinemia, suggesting that preoperative chemotherapy-induced splenic enlargement may be a caution for the occurrence of postoperative liver failure after major liver resection. The limitations of this study are the number of patients and lack of the evaluation of liver function after liver resection. Although previous report showed the discrepancy of the volume and the function of remnant liver [37], the results of the current study may be helpful for considering continuation of chemotherapy or operative risk after conversion surgery to avoid occurrence of liver dysfunction after hepatectomy.

\section{Conclusions}

Long-term oxaliplatin-based chemotherapy caused an increase in splenic enlargement. The splenic enlargement may cause impaired liver regeneration after hepatectomy and is likely to be related to occurrence of postoperative liver failure. The ratio of splenic volume before and after chemotherapy may be a useful indicator for decision-making of treatment strategy for unresectable CRLMs.

\section{Abbreviations}

CRLM: Colorectal liver metastases; VEGF: Vascular endothelial growth factor; MD-CT: Multidetector computed tomography; ELV: Entire liver volume; FRLV: Future remnant liver volume; RLV: Remnant liver volume;

ROC: Receiver-operating characteristic; EGFR: Epidermal growth factor receptor; BMI: Body mass index; ICG: Indocyanine green

Acknowledgements

Not applicable.

\section{Authors' contributions}

$T K, H Y$, and OM contributed to the study design. TK, HY, HS, HY, and KF contributed to the data acquisition. TK, HY, HS, HY, and KF contributed to the analysis and interpretation. TK and HY drafted the manuscript. HY, MM, and $\mathrm{OM}$ critically revised the manuscript. All authors approved the final version of the manuscript for publication.

\section{Funding}

None

\section{Availability of data and materials}

The datasets used for this study are available from corresponding author on reasonable request.

\section{Ethics approval and consent to participate}

This study was approved by the Institutional Board of Chiba University (\#3150). All patients have given informed consent in accordance with the ethical standards.

\section{Consent for publication}

Not applicable.

\section{Competing interests}

The authors declare that they have no competing interests.

\section{Author details}

${ }^{1}$ Department of General Surgery, Chiba University Graduate School of Medicine, 1-8-1 Inohana, Chuo-Ku, Chiba 260-8670, Japan. ²Department of Surgery, Oami Municipal Hospital, 884-1 Tomida, Oami-Shirasato-shi, Chiba 299-3221, Japan. ${ }^{3}$ Surgery and Digestive Disease Center, International University of Health and Welfare, Mita Hospital, 1-4-3 Mita, Minato-Ku, Tokyo 108-8329, Japan.

Received: 31 January 2020 Accepted: 15 June 2020

Published online: 23 June 2020

References

1. Kopetz S, Chang GJ, Overman MJ, Eng C, Sargent DJ, Larson DW, et al. Improved survival in metastatic colorectal cancer is associated with adoption of hepatic resection and improved chemotherapy. Journal of clinical oncology : official journal of the American Society of Clinical Oncology. 2009:27(22):3677-83.

2. Tournigand C, Andre T, Achille E, Lledo G, Flesh M, Mery-Mignard D, et al. FOLFIRI followed by FOLFOX6 or the reverse sequence in advanced colorectal cancer: a randomized GERCOR study. Journal of clinical oncology : official journal of the American Society of Clinical Oncology. 2004;22(2): 229-37.

3. Bokemeyer C, Bondarenko I, Hartmann JT, de Braud F, Schuch G, Zubel A et al. Efficacy according to biomarker status of cetuximab plus FOLFOX-4 as first-line treatment for metastatic colorectal cancer: the OPUS study. Annals of oncology: official journal of the European Society for Medical Oncology / ESMO. 2011;22(7):1535-46.

4. Folprecht $\mathrm{G}$, Gruenberger $\mathrm{T}$, Bechstein WO, Raab HR, Lordick F, Hartmann JT, et al. Tumour response and secondary resectability of colorectal liver metastases following neoadjuvant chemotherapy with cetuximab: the CELIM randomised phase 2 trial. Lancet Oncol. 2010;11(1):38-47.

5. Wong $R$, Cunningham D, Barbachano Y, Saffery C, Valle J, Hickish T, et al. A multicentre study of capecitabine, oxaliplatin plus bevacizumab as perioperative treatment of patients with poor-risk colorectal liver-only metastases not selected for upfront resection. Annals of oncology: official 
journal of the European Society for Medical Oncology / ESMO. 2011;22(9): $2042-8$.

6. Adam R, Delvart V, Pascal G, Valeanu A, Castaing D, Azoulay D, et al. Rescue surgery for unresectable colorectal liver metastases downstaged by chemotherapy: a model to predict long-term survival. Ann Surg. 2004; 240(4):644-57 discussion 57-8.

7. Zorzi D, Laurent A, Pawlik TM, Lauwers GY, Vauthey JN, Abdalla EK. Chemotherapy-associated hepatotoxicity and surgery for colorectal liver metastases. Br J Surg. 2007;94(3):274-86.

8. Nakano H, Oussoultzoglou E, Rosso E, Casnedi S, Chenard-Neu MP, Dufour $P$, et al. Sinusoidal injury increases morbidity after major hepatectomy in patients with colorectal liver metastases receiving preoperative chemotherapy. Ann Surg. 2008;247(1):118-24.

9. Overman MJ, Maru DM, Charnsangavej C, Loyer EM, Wang H, Pathak P, et al. Oxaliplatin-mediated increase in spleen size as a biomarker for the development of hepatic sinusoidal injury. Journal of clinical oncology : officia journal of the American Society of Clinical Oncology. 2010;28(15):2549-55.

10. Nagasue N, Yukaya H, Ogawa Y, Kohno H, Nakamura T. Human liver regeneration after major hepatic resection. A study of normal liver and livers with chronic hepatitis and cirrhosis. Ann Surg. 1987;206(1):30-9.

11. Garcea G, Maddern GJ. Liver failure after major hepatic resection. J HepatoBiliary-Pancreat Surg. 2009;16(2):145-55.

12. Nordlinger B, Van Cutsem E, Gruenberger T, Glimelius B, Poston G, Rougier $P$, et al. Combination of surgery and chemotherapy and the role of targeted agents in the treatment of patients with colorectal liver metastases: recommendations from an expert panel. Annals of oncology: official journal of the European Society for Medical Oncology / ESMO. 2009;20(6):985-92.

13. Dindo D, Demartines N, Clavien PA. Classification of surgical complications: a new proposal with evaluation in a cohort of 6336 patients and results of a survey. Ann Surg. 2004;240(2):205-13.

14. Boyce CJ, Pickhardt PJ, Kim DH, Taylor AJ, Winter TC, Bruce RJ, et al. Hepatic steatosis (fatty liver disease) in asymptomatic adults identified by unenhanced low-dose CT. AJR Am J Roentgenol. 2010;194(3):623-8.

15. Rahbari NN, Garden OJ, Padbury R, Brooke-Smith M, Crawford M, Adam R, et al. Posthepatectomy liver failure: a definition and grading by the International Study Group of Liver Surgery (ISGLS). Surgery. 2011;149(5):713-24.

16. Beppu T, Miyamoto Y, Sakamoto $Y$, Imai $K$, Nitta H, Hayashi $H$, et al. Chemotherapy and targeted therapy for patients with initially unresectable colorectal liver metastases, focusing on conversion hepatectomy and longterm survival. Ann Surg Oncol. 2014;21(Suppl 3):S405-13.

17. Adam R, Wicherts DA, de Haas RJ, Ciacio O, Levi F, Paule B, et al. Patients with initially unresectable colorectal liver metastases: is there a possibility of cure? Journal of clinical oncology : official journal of the American Society of Clinical Oncology. 2009;27(11):1829-35.

18. Lam WW, Spiro C, Laurence JM, Johnston E, Hollands MJ, Pleass HC, et al. A systematic review of clinical response and survival outcomes of downsizing systemic chemotherapy and rescue liver surgery in patients with initially unresectable colorectal liver metastases. Ann Surg Oncol. 2012;19(4):1292-301.

19. Karoui M, Penna C, Amin-Hashem M, Mitry E, Benoist S, Franc B, et al. Influence of preoperative chemotherapy on the risk of major hepatectomy for colorectal liver metastases. Ann Surg. 2006;243(1):1-7.

20. Robinson SM, Wilson CH, Burt AD, Manas DM, White SA. Chemotherapy associated liver injury in patients with colorectal liver metastases: a systematic review and meta-analysis. Ann Surg Oncol. 2012;19(13):4287-99.

21. Vauthey JN, Pawlik TM, Ribero D, Wu TT, Zorzi D, Hoff PM, et al. Chemotherapy regimen predicts steatohepatitis and an increase in 90-day mortality after surgery for hepatic colorectal metastases. Journal of clinical oncology : official journal of the American Society of Clinical Oncology. 2006:24(13):2065-72.

22. Kishi Y, Zorzi D, Contreras CM, Maru DM, Kopetz S, Ribero D, et al. Extended preoperative chemotherapy does not improve pathologic response and increases postoperative liver insufficiency after hepatic resection for colorectal liver metastases. Ann Surg Oncol. 2010;17(11):2870-6.

23. Imai K, Emi Y, lyama Kl, Beppu T, Ogata Y, Kakeji Y, et al. Splenic volume may be a useful indicator of the protective effect of bevacizumab against oxaliplatin-induced hepatic sinusoidal obstruction syndrome. European journal of surgical oncology : the journal of the European Society of Surgical Oncology and the British Association of Surgical Oncology. 2014; 40(5):559-66.

24. Ribero D, Wang H, Donadon M, Zorzi D, Thomas MB, Eng C, et al. Bevacizumab improves pathologic response and protects against hepatic injury in patients treated with oxaliplatin-based chemotherapy for colorectal liver metastases. Cancer. 2007:110(12):2761-7.

25. Hasselgren K, Malago M, Vyas S, Campos RR, Brusadin R, Linecker M, et al. Neoadjuvant chemotherapy does not affect future liver remnant growth and outcomes of associating liver partition and portal vein ligation for staged hepatectomy. Surgery. 2017;161(5):1255-65.

26. Inoue Y, Fujii K, Tashiro K, Ishii M, Masubuchi S, Yamamoto M, et al. Preoperative chemotherapy may not influence the remnant liver regenerations and outcomes after hepatectomy for colorectal liver metastasis. World J Surg. 2018;42(10):3316-30.

27. Simoneau E, Alanazi R, Alshenaifi J, Molla N, Aljiffry M, Medkhali A, et al. Neoadjuvant chemotherapy does not impair liver regeneration following hepatectomy or portal vein embolization for colorectal cancer liver metastases. J Surg Oncol. 2016;113(4):449-55.

28. Kang LI, Mars WM, Michalopoulos GK. Signals and cells involved in regulating liver regeneration. Cells. 2012;1 (4):1261-92.

29. Ding BS, Nolan DJ, Butler JM, James D, Babazadeh AO, Rosenwaks Z, et al. Inductive angiocrine signals from sinusoidal endothelium are required for liver regeneration. Nature. 2010;468(7321):310-5.

30. Wakiya T, Kudo D, Toyoki Y, Ishido K, Kimura N, Narumi S, et al. Evaluation of the usefulness of the indocyanine green clearance test for chemotherapyassociated liver injury in patients with colorectal cancer liver metastasis. Ann Surg Oncol. 2014;21(1):167-72.

31. Shan YS, Hsieh YH, Sy ED, Chiu NT, Lin PW. The influence of spleen size on liver regeneration after major hepatectomy in normal and early cirrhotic liver. Liver international : official journal of the International Association for the Study of the Liver. 2005;25(1):96-100.

32. Lee $\mathrm{SC}$, Jeong $\mathrm{HJ}$, Choi BJ, Kim SJ. Role of the spleen in liver regeneration in relation to transforming growth factor-beta1 and hepatocyte growth factor. J Surg Res. 2015;196(2):270-7.

33. Ueda S, Yamanoi A, Hishikawa Y, Dhar DK, Tachibana M, Nagasue N. Transforming growth factor-beta1 released from the spleen exerts a growth inhibitory effect on liver regeneration in rats. Laboratory investigation; a journal of technical methods and pathology. 2003;83(11):1595-603.

34. Schiffer E, Frossard JL, Rubbia-Brandt L, Mentha G, Pastor CM. Hepatic regeneration is decreased in a rat model of sinusoidal obstruction syndrome. J Surg Oncol. 2009;99(7):439-46.

35. Narita M, Oussoultzoglou E, Chenard MP, Rosso E, Casnedi S, Pessaux P, et al. Sinusoidal obstruction syndrome compromises liver regeneration in patients undergoing two-stage hepatectomy with portal vein embolization. Surg Today. 2011;41(1):7-17.

36. Simpson AL, Leal JN, Pugalenthi A, Allen PJ, DeMatteo RP, Fong Y, et al. Chemotherapy-induced splenic volume increase is independently associated with major complications after hepatic resection for metastatic colorectal cancer. J Am Coll Surg. 2015;220(3):271-80.

37. Truant $\mathrm{S}$, Baillet $\mathrm{C}$, Fulbert M, Olivier A, Sergent $\mathrm{G}$, Turpin $\mathrm{A}$, et al. Asymmetric kinetics of volume and function of the remnant liver after major hepatectomy as a key for postoperative outcome - a case-matched study. HPB : the official journal of the International Hepato Pancreato Biliary Association. 2019

\section{Publisher's Note}

Springer Nature remains neutral with regard to jurisdictional claims in published maps and institutional affiliations.

Ready to submit your research? Choose BMC and benefit from:

- fast, convenient online submission

- thorough peer review by experienced researchers in your field

- rapid publication on acceptance

- support for research data, including large and complex data types

- gold Open Access which fosters wider collaboration and increased citations

- maximum visibility for your research: over $100 \mathrm{M}$ website views per year

At $\mathrm{BMC}$, research is always in progress.

Learn more biomedcentral.com/submission 